\title{
Demographics of Thoracolumbar Fracture in Indian Population Presenting to a Tertiary Level Trauma Centre
}

\author{
Ketan Khurjekar, Shailesh Hadgaonkar, Ajay Kothari, Rishikesh Raut, \\ Vibhu Krishnan, Ashok Shyam, Parag Sancheti \\ Department of Spine Surgery, Sancheti Institute for Orthopaedics and Rehabilitation, Pune, India
}

Study Design: Prospective, cross-sectional, observational study.

Purpose: Spine traumata are devastating injuries, which may result in serious disabilities and dire consequences. The current study involves a detailed analysis and description of patients, who were operated at a tertiary care, urban level 1 Spine Centre in India. Overview of Literature: Various studies in literature have discussed the epidemiology and patterns of these injuries in trauma patients. However, literature describing the demographic profile and distribution of these traumata in the Indian population is scarce. Methods: The current study was conducted as a prospective trial involving patients, who were treated at our Spine Centre in India between July 2009 to December 2012. We studied 92 patients with thoraco-lumbar spine fracture, who were operated with short or long segment posterior stabilization. Epidemiological details, pre- and post-hospitalisation care received and other injury pattern factors were studied.

Results: Fall from height (46 patients, 50\%) was the most common mechanism observed in the patients. Sixty-three percent injuries belonged to $A 0$ type $A$ fractures, while $16.2 \%$ and $19.4 \%$ of the patients had suffered from $A 0$ types $B$ and $C$ injuries, respectively. Conclusions: We identified interesting epidemiological data and prevailing inadequacies in Emergency Spine care management in the study patients. These observations could facilitate implementation of the changes required to improve current standards of patient care.

Keywords: Spinal fractures; Thoracic vertebrae injuries; Demography; Trauma center; India

\section{Introduction}

Spine traumata are devastating injuries, which might result in serious disabilities and dire consequences, especially if managed inappropriately [1]. Despite significant improvements in the understanding and management of these injuries, the prognosis remains poor in a proportion of patients with significantly impaired neurological status
[2]. These injuries commonly occur in young males after high energy injuries; although the overall incidence actually increases with age consequent to bone insufficiencies secondary to osteoporosis and decreased bone mineral densities in the aging population [3].

Traumatic spinal cord injury occurs in an estimated 29-50 cases per million populations per year worldwide [4]. Thoraco-lumbar injuries constitute the major

Received Mar 24, 2014; Revised Nov 23, 2014; Accepted Nov 25, 2014

Corresponding author: Ashok Shyam

Sancheti Institute for Orthopaedics and Rehabilitation, 16, Shivajinagar, Pune 411 005, India

Tel: +91-9833110366, Fax: +91-2028999999, E-mail: doc.ashokshyam@gmail.com 
proportion of traumata involving the spine; and the management of these fractures involves various, available stabilisation modalities and techniques. Appropriate management options depend on multiple factors, including the morphology of fracture, patient profile, available infrastructure and surgical expertise [5-8].

Various studies in the literature have discussed the epidemiology and patterns of these injuries in trauma patients. However, literature describing the demographic profile and distribution of these traumata in the Indian population is scarce [3]. The current study involved a detailed analysis and description of patients, who were operated at a tertiary care, urban level 1 Spine Centre in India. We performed complete evaluation of all factors that might influence fracture healing, appropriate management protocols and prognosis in the patients. A discursive analysis of the issues involved against a backdrop of healthcare in a developing country was presented.

\section{Materials and Methods}

The current study was conducted as a prospective trial involving patients, who were admitted and treated at our Spine Centre in India during the period of July 2009 to December 2012. We studied a total of 92 patients with thoraco-lumbar spine fracture, who were operated with short or long segment posterior stabilization. The patients were received at the Emergency services and resuscitated in accordance with advanced trauma life support protocol. A thorough physical examination, including complete neurological examination was performed for all possible injuries. The patients were then administered urgent medical care, as required and the injuries were appropriately managed (including immobilisation, as required).

A detailed history, regarding the various aspects of their past health, social and economic issues, information of the current injury, prior treatment for the injury before hospitalisation, and mode of transportation employed for transfer, was obtained in all patients. The patients then underwent roentgenograms of chest and spine, computed tomography (CT) or magnetic resonance imaging scans of spine (as required), electrocardiogram and routine blood investigations. All epidemiological and technical data were analysed and presented.

Only patients with thoraco-lumbar fractures that needed to be surgically stabilised, were included after informed consent. Patients, who did not undergo surgery for their spine trauma and those who had undergone treatment for pathological fractures were excluded.

\section{Results}

Ninety-two patients were enrolled in the study. Of them, 82 were males and 10 were females ( $8: 1$ ratio). The mean age of our patients was 32 years (range, 18-59 years). Among the males, the mean age was 33 years (range, $18-59$ years), while the mean age among females was 31.5 years (range, 22-49 years).

The most common mechanism among the patients was fall from height (46 patients, 50\%). Of these, 18 patients (38\%) had a fall from a building, 11 patients $(25 \%)$ had sustained injury following fall from a tree, 8 patients had slipped into an unprotected well, 6 patients fell in a hilly terrain, and 3 patients fell while climbing stairs. The other most common mode of injury was road traffic accidents (43 patients, 46.7\%). Among these patients, the most common mechanism involved collision with another vehicle (19 patients, 44\%). The other modes included collision with a tree or other objects (16 patients, 37.2\%), slip and fall from a 2 wheeler ( 6 patients) and fall from the top of a running bus (2 patients). Three patients (3.3\%) had sustained injury following fall of a heavy object on their back. At the time of injury $23.9 \%$ (26 patients) patients were in an inebriated state. Four patients were road traffic accident victims involving death of at least 1 co-passenger.

The mean delay from the time of injury till presentation at our hospital was 16 days (ranging between 3 hours till 28 days). Most patients (58\%) were managed at $\geq 1$ lower level health care centre (private nursing homes in 13 patients, primary health center in 19 patients and private general trauma care hospitals in 21 patients). Six percent patients were managed at home for at least 24 hours before being taken to any medical care facility (Fig. 1). Lack of awareness of the critical nature of the injury and availability of specialised spine care was the cause of delay in 15\% patients. Eight percent patients were brought late due to misconceptions and fears of surgery. Financial constraints were crucial issues in $48 \%$ patients and caused delay in presentation to specialised spine centre. In $22 \%$ patients, the delay was secondary to availability of poor transport facilities and infrastructure and the distance from the centre (Fig. 2).

Among the patients who were referred from other medical care facilities, only 8 patients had received a 


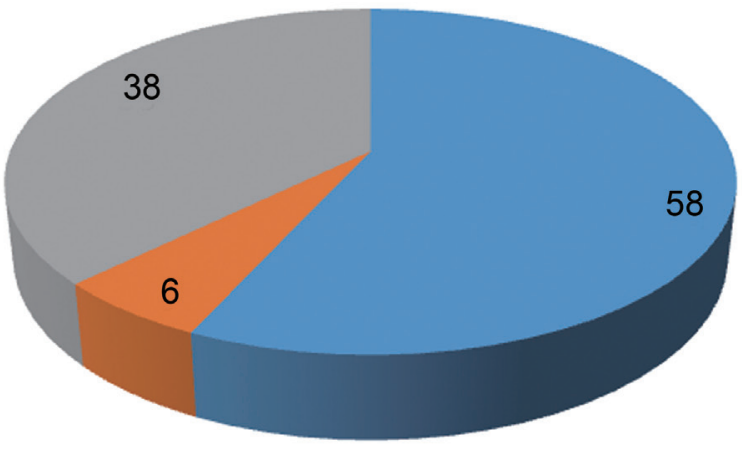

Patient managed at atleast one lower level care centre

Patients managed at home before being taken to any medical care facility

Patients brought directly to spine centre

Fig. 1. Percentage of patients receiving pre-hospital care.

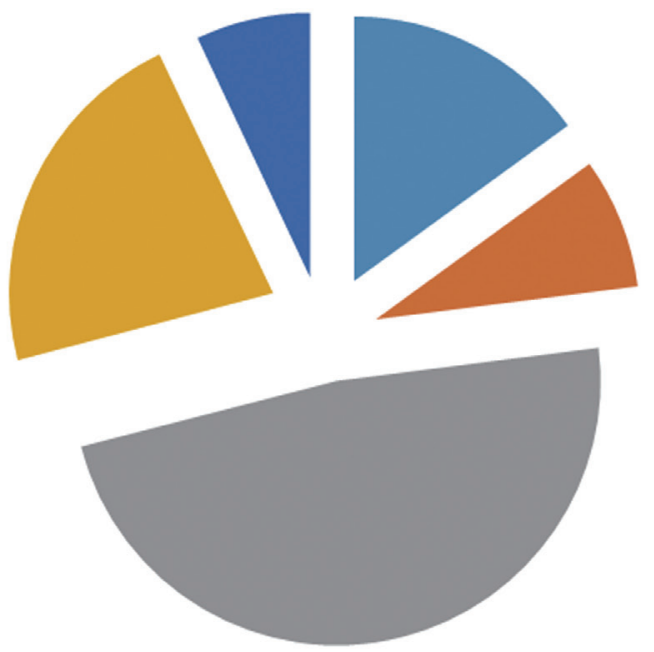

Lack of awareness

Misconceptions and fears regarding surgeries

Financial constraints

Poor transport facility

Others

Fig. 2. Reasons for delayed presentation.

dose of methylprednisolone sodium succinate within the initial 8 hours. Six patients, who had reached us within 8 hours, also received the recommended dosage. One patient was not given the recommended dose of steroids due to his diabetic status. Four patients in the series had neurological deterioration after being ambulatory at home or medical care centre, as a result of noncompliance with strict bed rest or gross initial under-evaluation of primary injury (patient neurological status was normal immediately post-trauma). Nevertheless, most patients (76\%) maintained strict bed rest.

The modality of transport, available to patients was another obvious deficiency. Only $16 \%$ of our patients had proper, strict immobilisation during transport after the injury (Fig. 3), mainly due to lack of awareness among the people involved in transferring such injured patients.

Majority of patients were involved in agricultural activities (36\%) prior to the injury, while other common occupations included private businesses (19\%), trading (11\%)

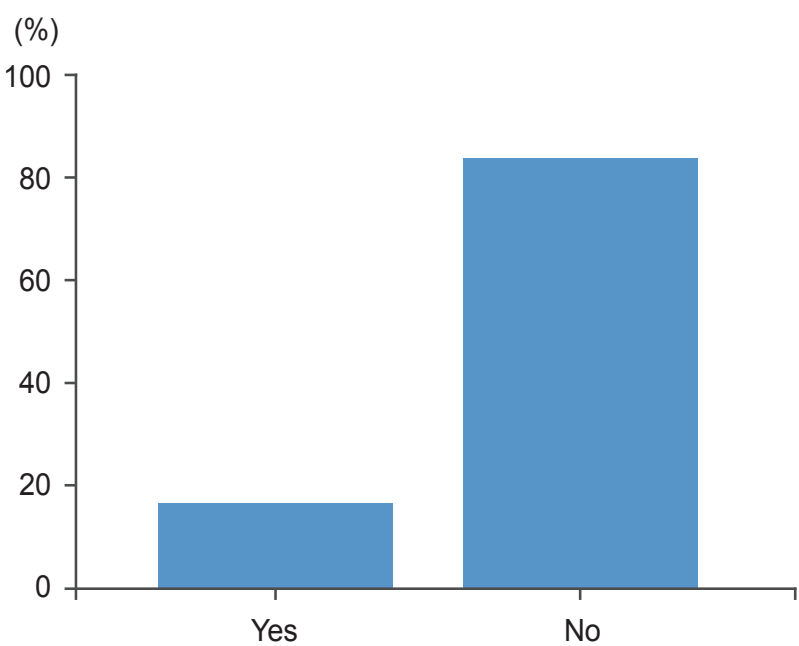

Fig. 3. Strict immobilisation during transport after the injury.

health-related (12\%), transport and construction jobs (15\%) and IT-sector (3\%). Three percent patients were 
unemployed or studying prior to injury (Fig. 4). Only $26 \%$ of patients belonged to the higher socioeconomic status population, while $35 \%$ of individuals belonged to the lower strata of the socioeconomic ladder (Fig. 5). Two patients had ankylosing spondylosis for which they were under regular treatment, while other common systemic co-morbidities were diabetes in 16 patients and hypertension in 23 patients.

The patient injury patterns were analysed and discussed. Thirty-one of the 92 patients included in the study had associated significant extremity injuries. While 13 had as-

(\%)

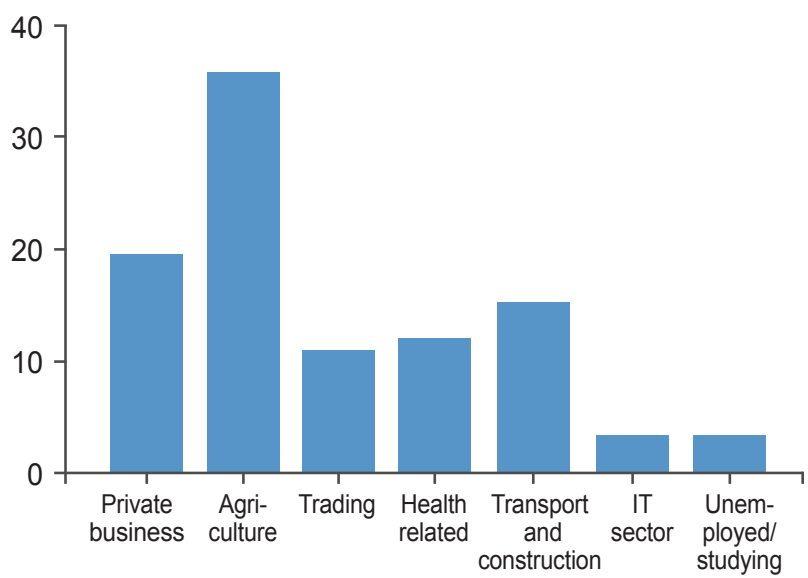

Activities

Fig. 4. Occupations of the patients prior to injury. sociated upper limb fractures, 22 had lower limb injuries (most common associated limb injuries noted were fractures involving the foot bones). Eight of the patients had associated head injuries, of which 1 had serious injury requiring craniotomy (Fig. 6). Most of the other patients required immediate head CT scan and were cleared after 24 hours observation. One patient had a serious blunt abdominal trauma which necessitated early laparotomy. Two patients had serious chest trauma, which was further treated with intercostal drainage.

The fracture levels in the thoracolumbar region of the

$$
\text { (\%) }
$$

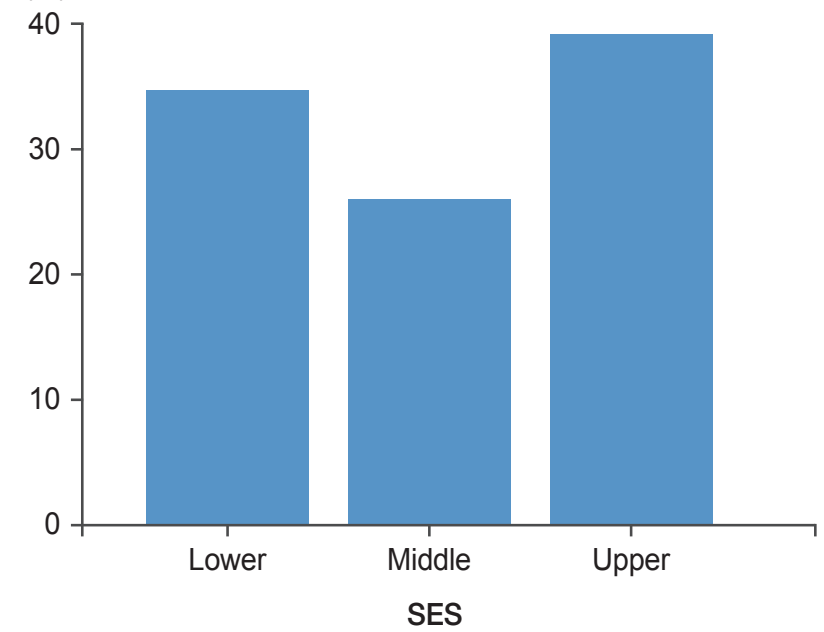

Fig. 5. Socio-economic status (SES) of patients.

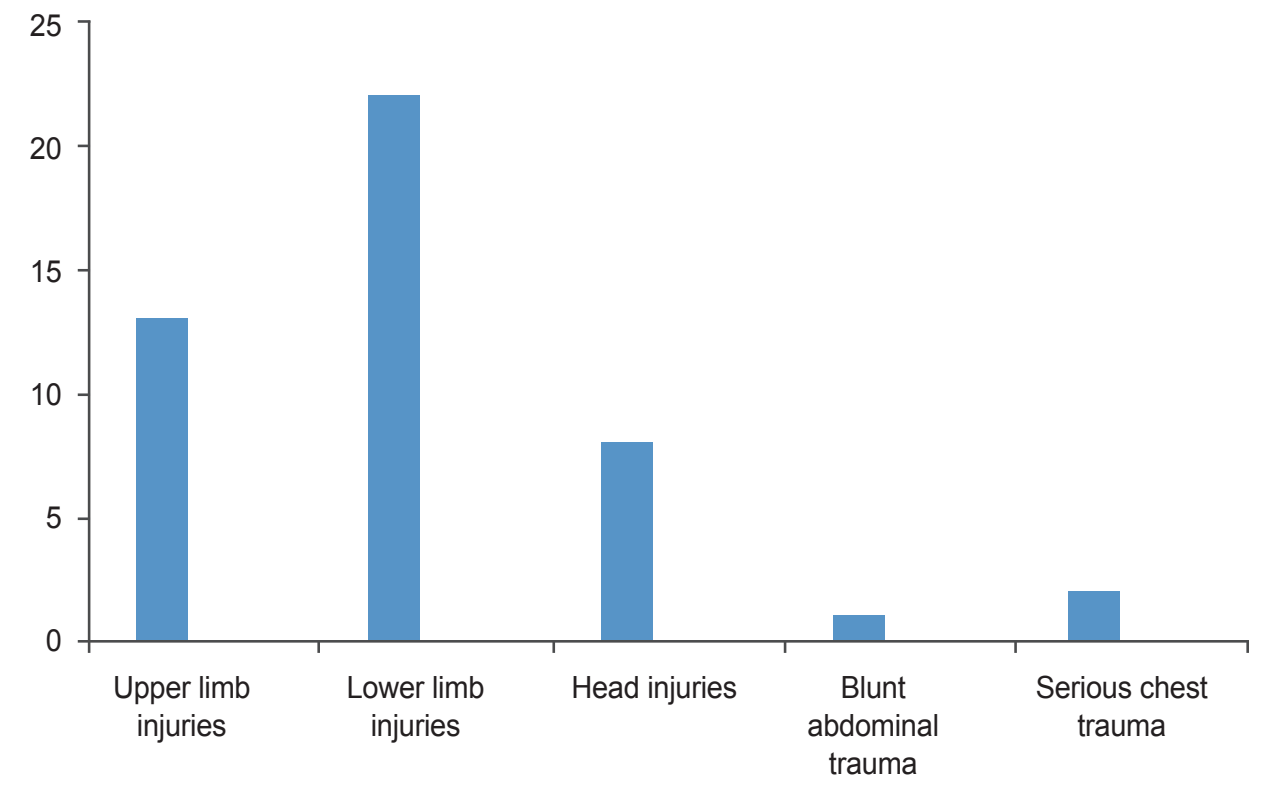

Fig. 6. Associated injury patterns. 
Table 1. Level of vertebral injury

\begin{tabular}{cccccc} 
& \multicolumn{3}{c}{ Vertebral level } \\
\cline { 2 - 5 } & D1-D4 & D5-D8 & D9-D11 & D12-L1 & L2-L5 \\
\hline
\end{tabular}

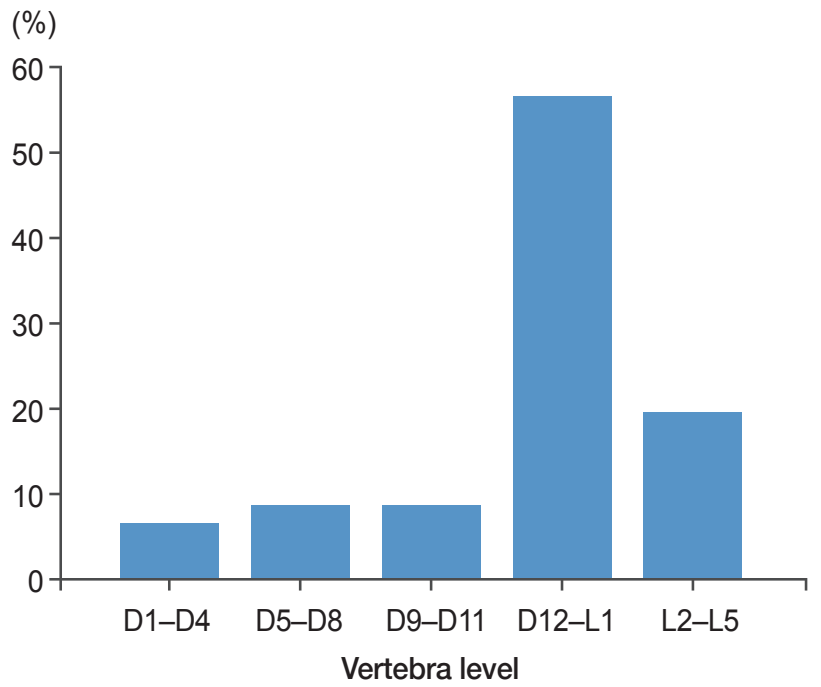

Fig. 7. Distribution of fracture levels involved.

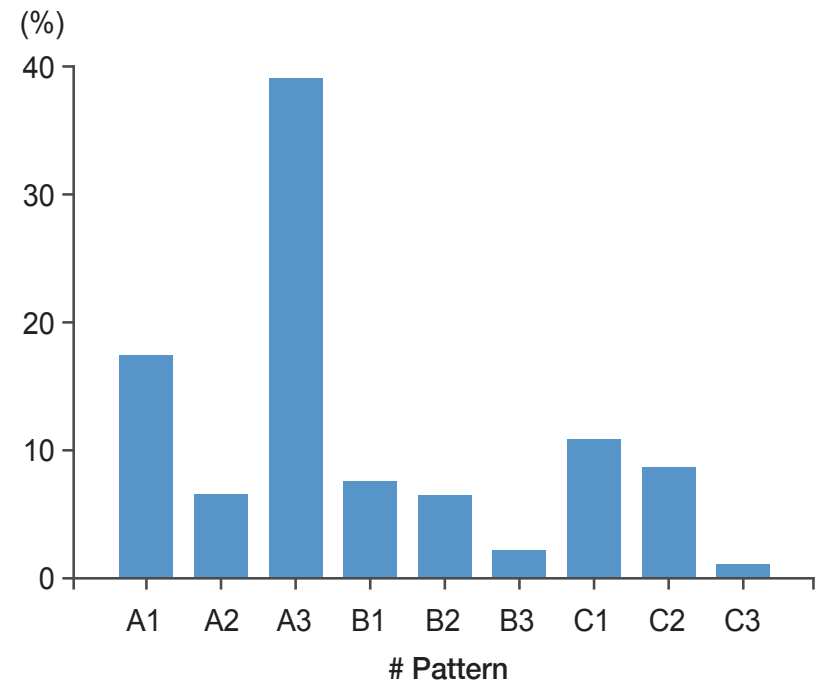

Fig. 8. Fracture patterns commonly observed.

Table 2. Fracture pattern observed as per A0 classification

\begin{tabular}{ccccccccccc} 
& \multicolumn{10}{c}{ Fracture pattern } \\
\cline { 2 - 12 } & A1 & A2 & A3 & B1 & B2 & B3 & C1 & C2 & C3 \\
No. of patients $(\%)$ & $16(17.4)$ & $6(6.5)$ & $36(39.1)$ & $7(7.6)$ & $6(6.5)$ & $2(2.1)$ & $10(10.8)$ & $8(8.6)$ & $1(1.08)$ \\
\hline
\end{tabular}

patients were shown below (Table 1, Fig. 7).

\section{Fracture patterns observed (Fig. 8)}

The fractures were classified in accordance with the AO classification described by Magerl et al. [9] and the distribution of fracture patterns was presented (Table 2).

The levels injured were analysed and tabulated (Table 3).

\section{Neurological status}

Eighty-eight patients in the study had some neurological involvement at presentation. The high incidence was due to the selective inclusion of patients who had undergone surgical stabilisation.

The neurological status of the patients (American spi-
Table 3. Distribution of multiple synchronous vertebral injuries

\begin{tabular}{lc} 
No. of levels & No. of patients $(\%)$ \\
\hline Single & $68(74)$ \\
Double & $14(15)$ \\
Triple & $8(9)$ \\
More than 3 levels & $2(2)$ \\
Non-contiguous & $4(4.34)$ \\
\hline
\end{tabular}

nal injuries association/Frankel grading) was tabulated below (Table 4, Fig. 9).

Of the 4 patients in the ASIA E group, 3 had AO type A1 injury, while 1 belonged to AO type A3. Thoracolumbar injury severity score (TLISS) scores in our patients were also analysed (Table 5). 
Table 4. Neurological statuses in injured patients

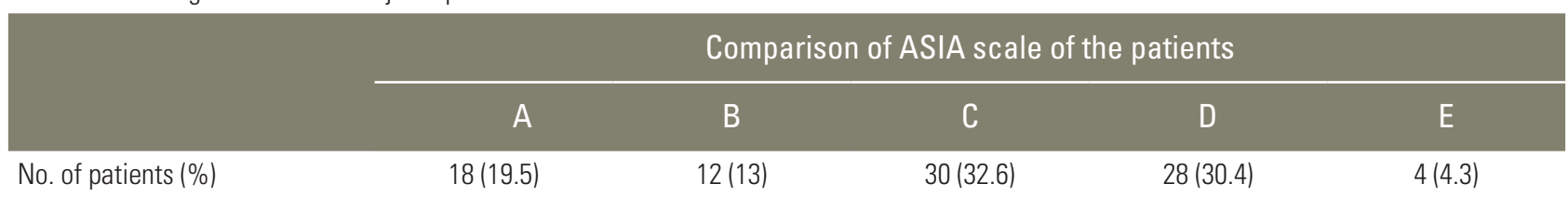

ASIA, American Spinal Injuries Association.

Table 5. Thoraco-lumbar injury severity score (TLISS) in injured patients

\begin{tabular}{cccccccc} 
& \multicolumn{7}{c}{ TLISS } \\
\cline { 2 - 7 } & 4 & 5 & 6 & 7 & 8 & 9 & 10 \\
No. of patients (\%) & 16 & 20 & 12 & 8 & 12 & 14 & 10 \\
\hline
\end{tabular}

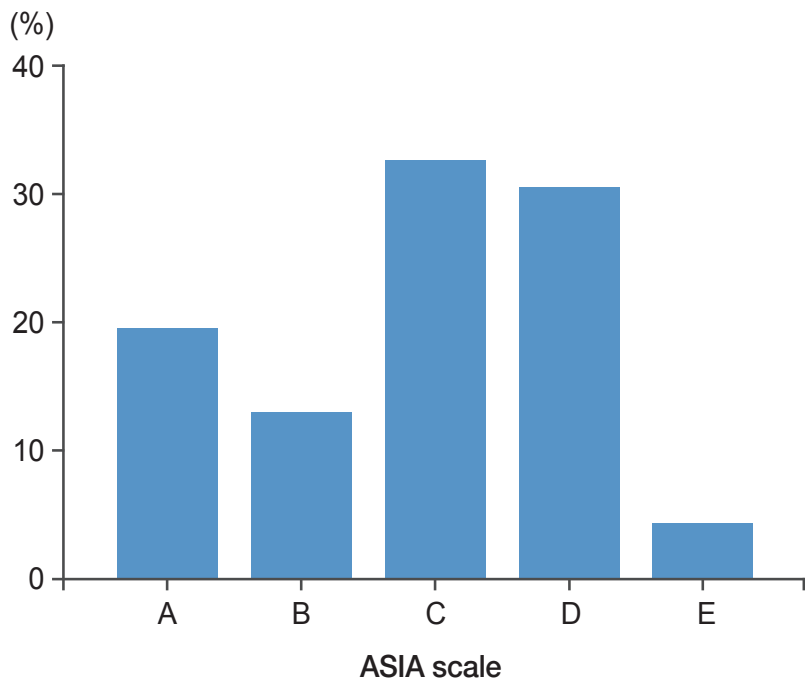

Fig. 9. Neurological statuses in injured patients. ASIA, American Spinal Injuries Association.

\section{Discussion}

Spine and spinal cord injuries are one of the most specialised areas of acute trauma management and health care delivery. These injuries have been of great interest in the past few decades and the management modalities have improved greatly, following advances in specialised medical and operative treatments. The management of these injuries largely depends on several factors, including demographic, socio-economic and infrastructural issues, apart from the general medical and injury-related patient status [10-14]. Trauma and Spine surgeons in the developing world are faced with various obstacles to appropriate health care delivery, however, there are relatively limited studies of the factors involved. We determined factors that might influence the management and outcome of patients, who were operated for significant spine trauma at an urban tertiary care spine centre in India.

We included only patients with severe spine injury who were managed surgically. The impact of the favorable or adverse factors was determined in this patient population. Our study suggested that young, adult males were the most susceptible to these high energy traumatic injuries. The existence of co-existing systemic morbidities was expectedly less (25\% prevalence), as in previous studies $[1,15,16]$.

The 2 most common injury mechanisms that comprised $96.7 \%$ injuries in our series included fall from height and road traffic accidents. Previous studies conducted in Jordan and Nigeria indicated that road traffic accidents and fall from height were the most common modes of spine injuries $[6,7,17]$. Bullet injuries and industrial accidents were other mechanisms of spine traumata $[3,6,7]$. We found that the injuries suffered in most patients were consequent to inadequate implementation of safety measures and non-compliance with safety proposals. This finding raises the need for creating awareness in our society, especially among the younger male population to ensure appropriate protective measures and prevent such devastating injuries.

Delay in presentation to a well-equipped spine centre is another concern [18-20]. In the UK, the average delay is estimated at 5.5 days [5]. Another study in Nigeria indicates a delay of 7 days [8]. A previous study in India showed a 45 day gross, mean delay in the hospital presentation of an injured spine patient [3]. There was a 16 day mean delay in our study. Delay in presentation has an im- 
portant association with the prognosis and final outcome of any spine trauma patient following surgery. Though lack of awareness, fears and misconceptions regarding spine trauma, lack of infrastructure and distance from the nearest spine centre were major factors, financial constraints faced by the patients was the foremost cause of delay in our study. More than one-third of the study patients belonged to the lower socioeconomic strata. This finding was of clinical relevance since these injuries can morbidly disable patients and result in long term absenteeism and rehabilitation. Furthermore, poorer populations might be prone to these injuries, due to lack of awareness and availability of proper safety measures.

Another important observation was that only $16 \%$ patients had access to proper transport facilities after spine trauma. We also observed that 4 patients had developed secondary cord damage after inadequate immobilisation, despite the lack of immediate neurological deficit on initial injury. This indicates lack of awareness concerning these injuries even among medical and paramedical health care workers. Medical care workers constitute an important component of the "transport and management chain" in these situations; and non-implementation of transfer norms has devastating consequences [21].

\section{Injury patterns}

Lower extremity injuries were the most common associated trauma, with foot injuries constituting a majority of these fractures in the series. Other studies indicated that pelvic fractures and lower limb injuries were the most commonly associated injuries in these situations [1,3,6$8,10]$. We also observed non-contiguous spine fractures in 2 patients. Twenty-six percent patients suffered from injuries to $\geq 1$ vertebral level. This reflects extremely high energy trauma and calls for high vigilance of the specialists during their assessment. Sixty-three percent injuries belonged to AO type A fractures, while $16.2 \%$ and $19.4 \%$ patients suffered from $\mathrm{AO}$ types $\mathrm{B}$ and $\mathrm{C}$ injuries, respectively.

\section{Neurological status}

There is a $21.2 \%$ reported incidence of neurological injury in patients with thoraco-lumbar fractures. Magerl et al. [9] reported a similar incidence (22\%) of neurological impairment associated with these injuries, although rates as high as $45 \%$ have also been reported in certain series. There is a 5 fold greater incidence of neurological deficit in males, as compared to females $[1,9,15,16]$, following thoraco-lumbar fractures. The current series only included patients who required surgical stabilisation for their spine fractures, hence the incidence of neurological impairment was as high as $88 \%$. Injuries with highest risk of neurological deficit include, fracture-dislocation (75\%) and burst fractures $(35 \%)$. Gertzbein $[15,16]$ showed that $19 \%$ of the thoraco-lumbar fracture patients suffered from a complete neurological deficit (categorised as the ASIA A group). Chung [1] and Pandey et al. [3] reported that $22.7 \%$ and $85 \%$ patients with neurological deficit, respectively, had a complete impairment (ASIA A). In our series, $19.5 \%$ patients had complete deficit (ASIA A) and $76 \%$ had incomplete neurological deficit (13\%-ASIA B, 32.6\%-ASIA C, and 30.4\%-ASIA D) resulting from the trauma. Patients with incomplete neurological deficit require greater attention and earlier surgical intervention to prevent possible, further deterioration and restore neurological functions secondary to reversible insults.

Only those patients with high TLISS (of at least 4-determined by the fracture pattern, neurological deficits, intactness of posterior ligament complex), who are typical candidates for surgical intervention, were included in our study. The study was conducted in a tertiary care Spine Centre in one of the bigger cities, which serves as one of the main referral centres for a major part of the population in Western India. The demographic details therefore represent general trends prevailing in the country. The epidemiological data and apparent inadequacies in emergency spine care management were further evaluated.

The current data could potentially facilitate the implementation of changes for improved patient care. Creating an awareness among the general population and medical and non-medical personnel involved in patient management at various stages is needed to improve health care during such emergencies.

\section{Conclusions}

We observed a large number of patients with serious spine injuries who required emergency care and specialised management protocols (including surgery). The infrastructure in developing nations is improving gradually and such injuries place a significant strain on available resources. Steps to prevent spinal injuries, strengthen 
patient transportation networks, increase the number and infrastructure of tertiary spinal trauma units, and implementation of multidisciplinary, comprehensive spine rehabilitation services might advance spine trauma care.

\section{Conflict of Interest}

No potential conflict of interest relevant to this article was reported.

\section{References}

1. Chung OM. Epidemiology of acute thoracolumbar fractures of young adults in Hong Kong. Hong Kong J Orthop Surg 2001;5:40-6.

2. Anderson PA, Rivara FP, Maier RV, Drake C. The epidemiology of seatbelt-associated injuries. J Trauma 1991;31:60-7.

3. Pandey V, Nigam V, Goyal TD, Chhabra H. Care of post-traumatic spinal cord injury patients in India: an analysis. Indian J Orthop 2007;41:295-9.

4. Vaccaro AR, Zeiller SC, Hulbert RJ, et al. The thoracolumbar injury severity score: a proposed treatment algorithm. J Spinal Disord Tech 2005;18:209-15.

5. Amin A, Bernard J, Nadarajah R, Davies N, Gow F, Tucker S. Spinal injuries admitted to a specialist centre over a 5-year period: a study to evaluate delayed admission. Spinal Cord 2005;43:434-7.

6. Nwadinigwe CU, Iloabuchi TC, Nwabude IA. Traumatic spinal cord injuries (SCI): a study of 104 cases. Niger J Med 2004;13:161-5.

7. Otom AS, Doughan AM, Kawar JS, Hattar EZ. Traumatic spinal cord injuries in Jordan: an epidemiological study. Spinal Cord 1997;35:253-5.

8. Solagberu BA. Spinal cord injuries in Ilorin, Nigeria. West Afr J Med 2002;21:230-2.

9. Magerl F, Aebi M, Gertzbein SD, Harms J, Nazarian S. A comprehensive classification of thoracic and lumbar injuries. Eur Spine J 1994;3:184-201.

10. Anderson S, Biros MH, Reardon RF. Delayed diag- nosis of thoracolumbar fractures in multiple-trauma patients. Acad Emerg Med 1996;3:832-9.

11. DeWald RL. Burst fractures of the thoracic and lumbar spine. Clin Orthop Relat Res 1984;(189):150-61.

12. Dai LY, Yao WF, Cui YM, Zhou Q. Thoracolumbar fractures in patients with multiple injuries: diagnosis and treatment-a review of 147 cases. J Trauma 2004; 56:348-55.

13. Burke DC, Murray DD. The management of thoracic and thoraco-lumbar injuries of the spine with neurological involvement. J Bone Joint Surg Br 1976;58:728.

14. Braakman R, Fontijne WP, Zeegers R, Steenbeek JR, Tanghe HL. Neurological deficit in injuries of the thoracic and lumbar spine: a consecutive series of 70 patients. Acta Neurochir (Wien) 1991;111:11-7.

15. Gertzbein SD. Fractures of the thoracic and lumbar spine. Baltimore: Williams \& Wilkins; 1992.

16. Gertzbein SD. Scoliosis Research Society. Multicenter spine fracture study. Spine (Phila Pa 1976) 1992;17: 528-40.

17. Surkin J, Gilbert BJ, Harkey HL 3rd, Sniezek J, Currier M. Spinal cord injury in Mississippi: findings and evaluation, 1992-1994. Spine (Phila Pa 1976) 2000;25: 716-21.

18. Scivoletto G, Morganti B, Molinari M. Early versus delayed inpatient spinal cord injury rehabilitation: an Italian study. Arch Phys Med Rehabil 2005;86:512-6.

19. Kishan S, Vives MJ, Reiter MF. Timing of surgery following spinal cord injury. J Spinal Cord Med 2005;28:11-9.

20. Tator CH, Duncan EG, Edmonds VE, Lapczak LI, Andrews DF. Neurological recovery, mortality and length of stay after acute spinal cord injury associated with changes in management. Paraplegia 1995; 33:254-62.

21. Prabhaka MM, Thakker TH. A follow-up program in India for patients with spinal cord injury: paraplegia safari. J Spinal Cord Med 2004;27:260-2. 